\title{
Thickness-Dependent Oscillations of the Critical Magnetic Field Induced by the Quantum Size Effect in Superconducting Nanofilms
}

\author{
P. Wójcik
}

Received: 9 June 2014 / Accepted: 7 August 2014 / Published online: 27 August 2014

(C) The Author(s) 2014. This article is published with open access at Springerlink.com

\begin{abstract}
The superconductor to normal metal transition driven by the in-plane magnetic field is investigated for $\mathrm{Al}$ nanofilms. Taking the Pauli breaking mechanism (with no orbital effect) into account, it is shown that the critical field oscillates as a function of the nanofilm thickness. This effect is discussed in terms of the quantization of electron energy caused by the confinement of its motion in the direction perpendicular to the film. The analysis of the critical field in the context of Clogston-Chandrasekhar limit is also included.
\end{abstract}

Keywords Superconducting nanofilms .

Quantum size effect · Critical field

\section{Introduction}

The superconductivity in nanoscale systems strongly deviates form that observed in the bulk. One of the most prominent manifestation of the interplay between the quantum size effect and the superconductivity was predicted in 1963 by Blatt and Thompson [4]. The authors of Ref. [4] studied superconducting properties of metallic nanofilms and found that the energy gap oscillates as a function of the nanofilm thickness. It was shown that this phenomena is directly related to the quantization of the electron energy resulting from the confinement of its motion in the direction perpendicular to the film. Due to technological difficulties in the preparation of uniform metallic films (which are typically polycrystalline and contain a large number of

P. Wójcik ( $\square)$

Faculty of Physics and Applied Computer Science, AGH

University of Science and Technology,

al. Mickiewicza 30, Kraków, Poland

e-mail: pawel.wojcik@fis.agh.edu.pl defects), the experimental confirmation of the thicknessdependent energy gap oscillations has been reported only recently [10]. In Ref. [10], the oscillations of the critical temperature as a function of the number of atomic monolayers have been found for ultra-thin $\mathrm{Pb}$ films grown on $\mathrm{Si}(111)$ substrate. This experiment reopens an extensive research concerning the interplay of the superconductivity and the quantum confinement in nanoscale systems $[1,3$, $11,14,18,19,22]$. The study of the superconductivity in $\mathrm{Pb}$ nanofilms has been extended by Eom et al. [8]. By the use of the scanning gate microscopy, $T_{c}$ oscillations in the epitaxially grown crystalline $\mathrm{Pb}$ films have been measured in the thickness range $5--18$ ML [8]. Very recently, it has been observed the superconductivity in a single $\mathrm{Pb}$ and In atomic layer [20,23]. For Al nanofilms, the increase of the superconducting energy gap with decrease of the film thickness has been reported in Ref. [7]. Our recent theoretical calculations [21], which take into account an influence of the electron density and the surface roughness, allow to reconstruct experimental data [7] and show that the experimentally observed thickness dependence of the energy gap can be well fitted in the regime of the oscillatory behavior of the energy gap. Recently, the growing attention of the scientists has attracted the effect of the quantum confinement on the superconductor to normal metal transition induced by the magnetic field. It has been theoretically predicted [17] that decrease of the energy gap as a function of the magnetic field has the form of jumps which are related to depairing in the subsequent subbands. The oscillations of the perpendicular upper critical field in thin lead films have been reported by Bao et al. in Ref. [2]. An interesting effect has been also found in amorphous $\mathrm{Pb}$ nanofilms [9], for which the increase of the critical temperature after application of a parallel magnetic field has been observed. 
Motivated by these experiments, in the present paper, we investigate the superconductor-normal metal transition driven by the in-plane magnetic field. We show that the critical field oscillates as a function of the nanofilm thickness and is higher than the Clogston-Chandrasekhar paramagnetic limit $[5,6]$. We also discuss the thermal effect on the superconductor-normal metal transition. The present paper is organized as follows. In Section 2, we introduce the theoretical model based on the Bogoliubov-de Gennes equations. In Section 3, we discuss the results, while the summary is included in Section 4.

\section{Theoretical Method}

The spin-generalized Bogoliubov-de Gennes (BdG) equations have the form

$$
\left(\begin{array}{cccc}
H_{e}^{\uparrow} & \Delta & 0 & 0 \\
\Delta^{*} & -H_{e}^{\downarrow *}, & 0 & 0 \\
0 & 0 & H_{e}, & \Delta \\
0 & 0 & \Delta^{*} & -H_{e}^{\uparrow *}
\end{array}\right)\left(\begin{array}{c}
\mathcal{U}_{i}^{\uparrow} \\
\mathcal{V}_{i}^{\downarrow} \\
\mathcal{U}_{i}^{\downarrow} \\
-\mathcal{V}_{i}^{\uparrow}
\end{array}\right)=E_{i}\left(\begin{array}{c}
\mathcal{U}_{i}^{\uparrow} \\
\mathcal{V}_{i}^{\downarrow} \\
\mathcal{U}_{i}^{\downarrow} \\
-\mathcal{V}_{i}^{\uparrow}
\end{array}\right),
$$

where $\mathcal{U}_{i}^{\sigma}$ and $\mathcal{V}_{i}^{\sigma}$ are the spin-dependent electron-like and hole-like wave functions $(\sigma=\uparrow \downarrow), \Delta$ is the superconducting energy gap, and $E_{i}$ is the quasi-particle energy. The Hamiltonian $H_{e}^{\sigma}$ is given by

$H_{e}^{\sigma}=\frac{1}{2 m}\left(-i \hbar \nabla-\frac{e}{c} \mathbf{A}\right)^{2}+s \mu_{B} H_{\|}-\mu_{F}$,

where $s= \pm 1$ corresponds to the spin $\sigma=\uparrow \downarrow, m$ is the free electron mass, $\mu_{B}$ is the Bohr magneton, $\mu_{F}$ is the chemical potential, and $\mathbf{A}$ is the vector potential.

In our calculations, we neglect the orbital effect and consider the superconducting nanofilms in the clean paramagnetic limit. This approximation is justified for the nanofilms with the thickness less than the magnetic length $a_{H}=$ $\sqrt{\hbar / e H_{\|}}$- the use of the Clogston-Chandrasekhar paramagnetic field for $\mathrm{Al}, H^{C C}=3 \mathrm{~T}$ gives $a_{H}=14.6 \mathrm{~nm}$. If we assume that the system is infinite in the $x-y$ plane $\left(L_{x}, L_{y} \rightarrow \infty\right)$, the quasi-particle wave function can be expressed as

$$
\left(\begin{array}{l}
\mathcal{U}_{k_{x} k_{y} v}^{\sigma}(\mathbf{r}) \\
\mathcal{V}_{k_{x} k_{y} v}^{\bar{\sigma}}(\mathbf{r})
\end{array}\right)=\frac{e^{i k_{x} x}}{\sqrt{L_{x}}} \frac{e^{i k_{y} y}}{\sqrt{L_{y}}}\left(\begin{array}{c}
u_{\nu}^{\sigma}(z) \\
v_{\nu}^{\bar{\sigma}}(z)
\end{array}\right)
$$

where $\bar{\sigma}$ denotes the opposite spin. In (3), the index $i$ has been replaced by $k_{x}, k_{y}, v$, where $k_{x}$ and $k_{y}$ are the free electron wave vector components in the $x$ and $y$ direction while $v$ labels the quantum well states in the $z$ direction. The order parameter $\Delta(z)$ is given by

$$
\begin{aligned}
\Delta(z) & =\frac{g}{2 \pi} \int d k_{\|} k_{\|} \sum_{\nu}\left\{u_{\nu}^{\uparrow}(z) v_{\nu}^{\downarrow *}(z)\left[1-f\left(E_{\nu}^{\uparrow}\right)\right]\right. \\
& \left.+u_{\nu}^{\downarrow}(z) v_{\nu}^{\uparrow *}(z) f\left(E_{\nu}^{\downarrow}\right)\right\},
\end{aligned}
$$

where $g$ is the electron-phonon coupling and $f(E)$ is the Fermi-Dirac distribution. The summation in (4) is carried out only over these states for which the single-electron energy $\xi_{k_{x} k_{y} v}^{\sigma}$ satisfies the condition $\left|\xi_{k_{x} k_{y} v}^{\sigma}\right|<\hbar \omega_{D}$, where $\omega_{D}$ is the Debye frequency and

$$
\begin{aligned}
\xi_{k_{x} k_{y} \nu}^{\sigma} & =\int_{0}^{d} d z\left\{u_{v}^{\sigma *}(z) H_{e}^{\sigma}(z) u_{\nu}^{\sigma}(z)\right. \\
& \left.+v_{v}^{\bar{\sigma} *}(z) H_{e}^{\bar{\sigma}}(z) v_{v}^{\bar{\sigma}}(z)\right\},
\end{aligned}
$$

where $d$ is the nanofilm thickness. The system of (1)-(4) is solved in a self consistent manner until the convergence is reached. As a result, the spatially varying energy gap $\Delta(z)$ is obtained.

We define the spatially averaged energy gap as

$\bar{\Delta}=\frac{1}{d} \int_{0}^{d} \Delta(z) d z$.

For each nanofilm thickness, we determine the chemical potential by using the formula

$$
\begin{aligned}
n_{e} & =\frac{1}{\pi d} \int d k_{\|} k_{\|} \sum_{\sigma} \sum_{\nu} \int_{0}^{d} d z\left\{\left|u_{\nu}^{\sigma}(z)\right|^{2} f\left(E_{v}\right)\right. \\
& \left.+\left|v_{\nu}^{\bar{\sigma}}(z)\right|^{2}\left[1-f\left(E_{\nu}\right)\right]\right\} .
\end{aligned}
$$

In the calculations, we adopt the hard-wall potential profile as the boundary conditions in the $z$ direction.

\section{Results}

In this section, we discuss the superconductor to normal metal phase transition driven by the in-plane magnetic field. We consider $\mathrm{Al}$ nanofilms in the thickness range $1-5 \mathrm{~nm}$ which is less than $a_{H}=14.6 \mathrm{~nm}$ and guarantees that the paramagnetic approximation used in the paper is satisfied. The calculations have been carried out for the following values of the parameters: $g N_{\text {bulk }}(0)=0.18$ where $N_{\text {bulk }}(0)=$ $m k_{F} /\left(2 \pi^{2} \hbar^{2}\right)$ is the density of the single-electron states at the Fermi level, $\hbar \omega_{D}=32.31 \mathrm{meV}$ and the bulk energy gap $\Delta_{\text {bulk }}=0.25 \mathrm{meV}$. The Fermi level in the bulk $\mu_{\text {bulk }}$ is treated as a fitting parameter [16], and its value is determined based on the experimental results [7], $\mu_{\text {bulk }}=$ $0.9 \mathrm{eV}$.

In Fig. 1a, we present the critical in-plane magnetic field $H_{c, \|}$ as a function of the nanofilm thickness $d$ for 

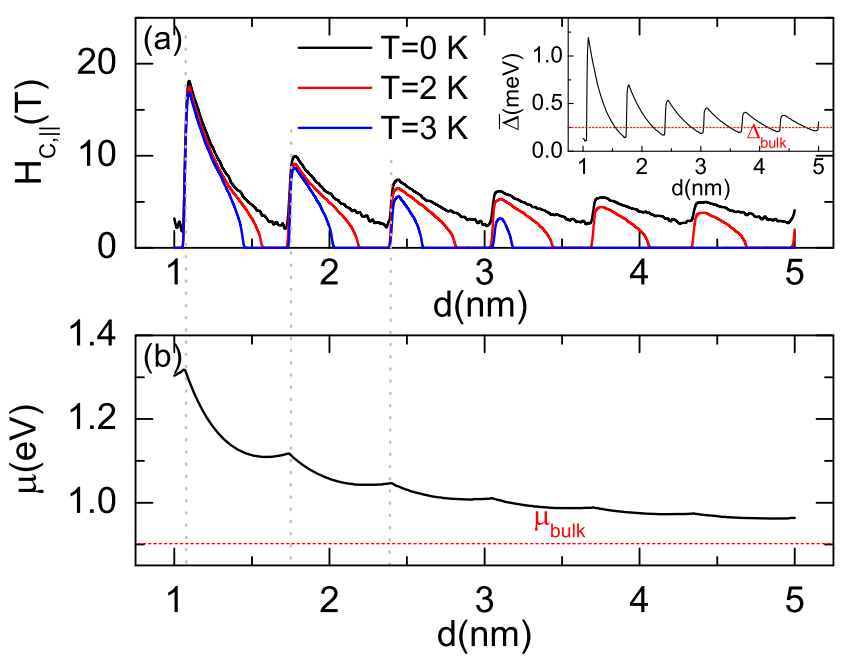

Fig. 1 a In-plane critical magnetic field $H_{c, \|}$ and $\mathbf{b}$ chemical potential $\mu$ as a function of the nanofilm thickness $d$. The inset presents the spatially averaged energy gap $\bar{\Delta}$ as a function of the thickness $d$

different temperatures. In our calculations, the value of $H_{c, \|}$ is defined as the field for which the spatially averaged energy gap $\bar{\Delta}$ drops below $0.01 \Delta_{b u l k}$. Figure 1a shows that the critical field oscillates as a function of the film thickness. It strongly increases for certain specific thicknesses and then exponentially decreases until the next enhancement of the critical field appears. The "toothlike" thickness-dependence of the critical field is directly related to the quantization of the electron energy resulting from the confinement of its motion in the direction perpendicular to the film. If the motion of electron in the $z$ direction is reduced to the nanometer scale, the Fermi sphere transforms into series of parabolic subbands whose energy decreases with increasing nanofilm thickness. Since the Cooper-pairing in the phonon-mediated superconductor is determined by the number of the electron states in the energy window $\left[\mu-\hbar \omega_{D}, \mu+\hbar \omega_{D}\right]$ ( $\hbar \omega_{D}$ is Debye energy), one can observe the enhancement of the energy gap each time, the subsequent subband passes through the Fermi surface. The spatially averaged energy gap as a function of the nanofilm thickness is presented in the inset of Fig. 1a. For ultra thin nanofilms in the parallel magnetic field depairing effects related to the orbital effect can be neglected and only the Pauli breaking mechanism determines the value of the critical field. Therefore, the oscillatory behavior of the energy gap induced by the quantum confinement results in the thickness-dependent oscillations of the critical field presented in Fig. 1a. In the bulk, the paramagnetic (Pauli) limit is given by Clogston-Chandrasekhar (CC) $[5,6]$ formula $-H^{C C}=\Delta_{\text {bulk }} /\left(\sqrt{2} \mu_{B}\right)$, which for $\mathrm{Al}$ is equal to $H^{C C}=3 \mathrm{~T}$. Note that the value of $H^{C C}$ is comparable to the calculated critical field only for nanofilms with the thickness for which $H_{c,||}$ reaches its minimum (see Fig. 1).
For majority part of the thickness range, the calculated critical field is several times higher than $H^{C C}$. Such observation for the $\mathrm{Pb}$ nanofilms has been recently reported in Ref. [15] in which the experimentally measured critical field $H_{C, \|}$ has been much higher than the paramagnetic limit $H^{C C}$. The discrepancy of the calculated critical field and $H^{C C}$ results from the deviation of the energy gap $\bar{\Delta}$ from its bulk value. It indicates that the Clogston-Chandrasekhar formula has to be reformulated and for nanofilms has the form $H^{C C}(d)=$ $\Delta_{v}(d) /\left(\sqrt{2} \mu_{B}\right)$, where $\Delta_{v}$ denotes the expectation value of energy gap in the superconducting subband $v$ which in our case does not depend of $v$ (Fig. 2). In order to show which of the subbands participate in the Cooper pair condensation, in Fig. 2, we present the quasi-particle energy $E$ as a function of the wave vector $k_{\|}=\sqrt{k_{x}^{2}+k_{y}^{2}}$ for nanofilm thicknesses corresponding to the first two maxima of the critical field from Fig. 1a.

We can see that for the nanofilm thickness $d=1.1 \mathrm{~nm}$ [Fig.2a] the enhancement of the critical field corresponds
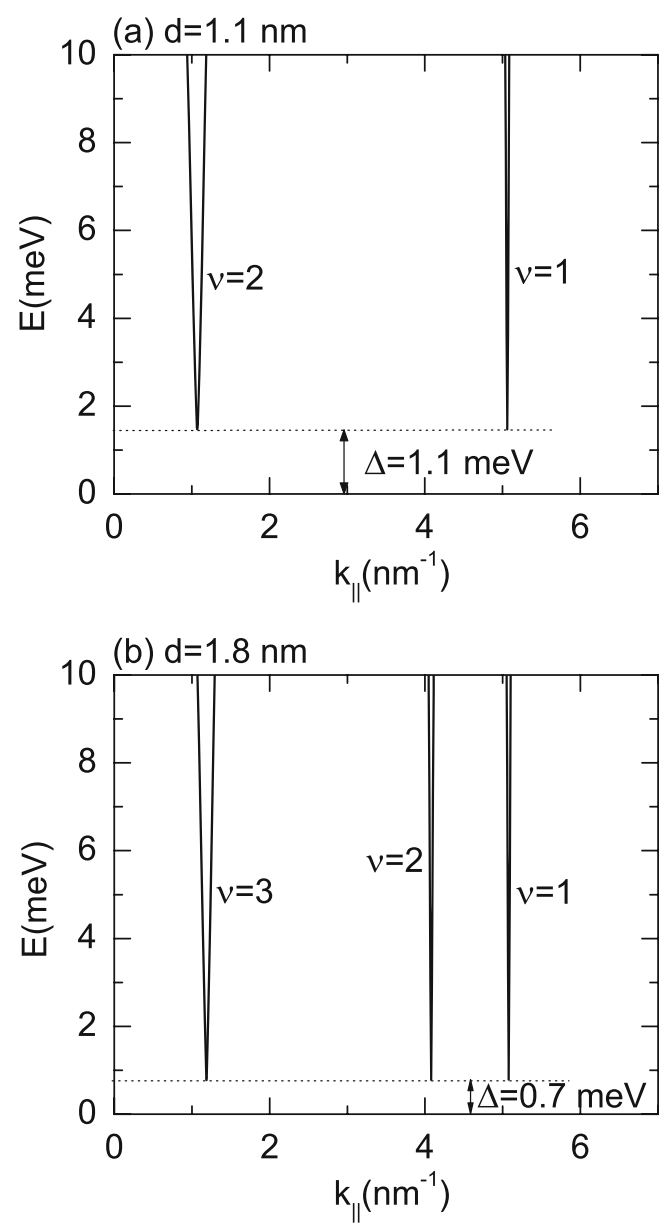

Fig. 2 Quasi-particle energy $E$ as a function of the wave vector $k_{\|}$ for the film thickness a $d=1.1 \mathrm{~nm}$ which corresponds to the first enhancement of the critical field and $\mathbf{b} d=1.8 \mathrm{~nm}$ which is related to the second enhancement of the critical field 
Fig. 3 (Color online) Spatially averaged energy gap $\bar{\Delta}$ as a nanofilm thicknesses a $d=1.1 \mathrm{~nm}, \mathbf{b} d=1.7 \mathrm{~nm}$, and c $d=1.8 \mathrm{~nm}$ which correspond to the first maxima, minima, and the second maxima of $H_{c, \|}$ in Fig. 1a. The first- to secondorder transition is marked by the dashed vertical line function of the magnetic field $H$ and temperature $T$ for the
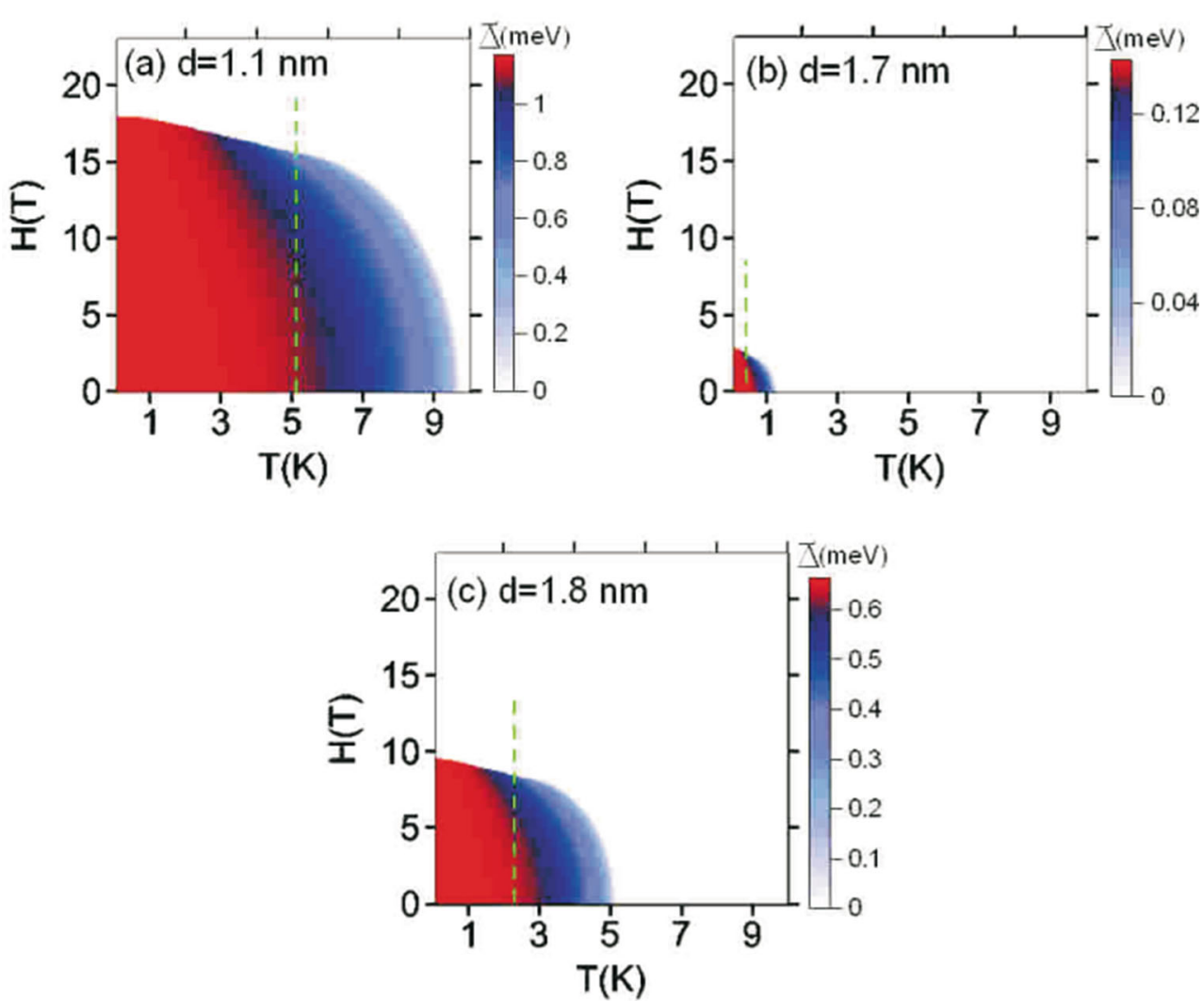

to the Cooper pairing of electrons from the subband $v=$ 2. By analogy, the analysis of Fig. $2 \mathrm{~b}$ allows to conclude that the second maxima corresponds to the condensation of electrons from the subband $v=3$. It should be also noted that the amplitude of $H_{c, \|}$ oscillations in Fig. 1a decreases with increasing nanofilm thickness and converges to their bulk value $H^{C C}$ for sufficiently thick nanofilms.

To analyze the thermal effect in the superconductor to normal metal transition, in Fig. 3, we present the spatially averaged energy gap as a function of the magnetic field and temperature. The results in Fig. 3 have been calculated for the nanofilm thicknesses (a) $d=1.1 \mathrm{~nm}$ which corresponds to the first maxima, (b) $d=1.7 \mathrm{~nm}$ corresponding to the minima, and (c) $d=1.8 \mathrm{~nm}$ which is related to the second maxima of $H_{c, \|}$ in Fig. 1a. We can see that the range of parameters $(H, T)$ for which the film exists in the superconducting state strongly depends on the nanofilm thickness. It has been found that the initial behavior of $H_{c, \|}(T)$ at the temperature close to $T_{c}$ can be well estimated by the formula $H_{c, \|}(T)=A H_{c, \|}(0)\left[1-T_{c}(T) / T_{c}(0)\right]^{1 / 2}$, where $H_{c, \|}(0)$ and $T_{c}(0)$ denote the zero temperature critical field and the critical temperature for $H=0$ while $A$ is a fitting parameter. Note that, in contrast to the orbital limit with no Pauli effect, the slope of $H_{c, \|}(T)$ at critical temperature $T_{c}$ is infinite. In this range, the transition at $H_{c}$, is of second order. In the Pauli limit, the second-order transition is suppressed with decreasing temperature and below $T_{c}(T) / T_{c}(0)=0.56$ it becomes the first-order [12, 13]. In Fig. 3, the first- to second-order transition is marked by the dashed vertical line.

\section{Summary}

The quantum size effect on the in-plane paramagnetic critical field is investigated with the use of the spin-generalized Bogoliubov-de Gennes equations. It is shown that the critical field oscillates as a function of the nanofilm thickness which is explained in terms of the electron energy quantization induced by the confinement in the direction perpendicular to the film. It is found that the zero-temperature critical field in the nanofilms tends to be higher than the ClogstonChandrasekhar paramagnetic limit which is in agreement with recent experiments [15]. The validity of the $\mathrm{CC}$ formula can be restored by the use of the thickness-dependent energy gap.

Acknowledgments This work is financed from the budget for Polish Science in the years 2013-2015. Project number: IP2012 048572. 
Open Access This article is distributed under the terms of the Creative Commons Attribution License which permits any use, distribution, and reproduction in any medium, provided the original author(s) and the source are credited.

\section{References}

1. Altomare, F., Chang, A.M., Melloch, M.R., Hong, Y., Tu, C.W.: Phys. Rev. Lett. 97, 017,001 (2006)

2. Bao, X.Y., Zhang, Y.F., Wang, Y., Jia, J.F., Xue, Q.K., Xie, X.C., Zhao, Z.X.: Phys. Rev. Lett. 95, 247, 005 (2005)

3. Bezryadin, A., Lau, C.N., Tinkham, M.: Nature 404, 971 (2000)

4. Blatt, J.M., Thompson, C.J.: Phys. Rev. Lett. 10, 332 (1963)

5. Chandrasekhar, B.S.: Appl. Phys. Lett. 1,7 (1962)

6. Clogstone, A.M.: Phys. Rev. Lett. 9, 266 (1962)

7. Court, N.A., Ferguson, A.J., Clark, R.G.: Supercond. Sci. Technol. 21, 015,013 (2007)

8. Eom, D., Qin, S., Chou, M.Y., Shih, C.K.: Phys. Rev. Lett. 96, 027,005 (2006)

9. Gardner, H.J., Kumar, A., Yu, L., Xiong, P., Warusawithana, M.P., Wang, L., Vafek, O., Schlom, D.G.: Nat. Phys. 7, 895 (2011)

10. Guo, Y., Zhang, Y.F., Bao, X.Y., Han, T.Z., Tang, Z., Zhang, L.X., Zhu, W.G., Wang, E.G., Niu, Q., Qiu, Z.Q., Jia, J.F., Zhao, Z.X., Xue, Q.K.: Science 306, 1915 (2004)
11. Janković, L., Gournis, D., Trikalitis, P.N., Arfaoui, I., Cren, T., Rudolf, P., Sage, M.H., Palstra, T.T.M., Kooi, B., De Hosson, J., Karakassides, A., Dimos, K., Moukarika, A., Bakas, T.: Nano Lett. 6, 1131 (2006)

12. Maki, K. Prog. Theor. Phys. 31, 945 (1964)

13. Maki, K.: Phys. Rev. 148, 362 (1966)

14. Savolainen, M., Toulboltsev, V., Koppinen, P., Riikonen, K.P., Arutyunov, K.: Appl. Phys. A: Mater. Sci. Process 79, 1769 (2004)

15. Sekihara, T., Masutomi, R., Okamato, T.: Phys. Rev. Lett. 111, 057,005 (2013)

16. Shanenko, A.A., Croitoru, M.D., Peeters, F.M.: Phys. Rev. B 75, 014,519 (2007)

17. Shanenko, A.A., Croitoru, M.D., Peeters, F.M.: Phys. Rev. B 78, 024,505 (2008)

18. Shanenko, A.A., Croitoru, M.D., Zgirski, M., Peeters, F.M., Arutyunov, K.: Phys. Rev. B 74, 052,502 (2006)

19. Tian, M.L., Wang, J.G., Kurtz, J.S., Liu, Y., Chan, M.H.W., Mayer, T.S., Mallouk, T.E.: Phys. Rev. B 71, 104,521 (2005)

20. Uchihashi, T., Mishra, P., Aono, M., Nakayama, T.: Phys. Rev. Lett. 107, 207,001 (2011)

21. Wójcik, P., Zegrodnik, M.: Phys. Status Solidi B (2014)

22. Zgirski, M., Riikonen, K.P., Touboltsev, V., Arutyunov, K.: Nano Lett. 5, 1029 (2005)

23. Zhang, T., Cheng, P., Li, W.J., Sun, Y.J., Wang G Zhu, X.G., He, K., Wang, L.L., Ma, X.C., Chen, X., Wang, Y.Y., Liu, Y., Jia, J.F., Xue, Q.K.: Q, L. H. Nat. Phys 6, 104 (2010) 